\title{
HERMIONE: a randomized Phase 2 trial of MM-302 plus trastuzumab versus chemotherapy of physician's choice plus trastuzumab in patients with previously treated, anthracycline-naïve, HER2-positive, locally advanced/metastatic breast cancer
}

Kathy Miller ${ }^{1}$, Javier Cortes ${ }^{2}$, Sara A. Hurvitz ${ }^{3}$, Ian E. Krop ${ }^{4}$, Debu Tripathy ${ }^{5}$, Sunil Verma ${ }^{6}$, Kaveh Riahi ${ }^{7}$, Joseph G. Reynolds ${ }^{7 *}$, Thomas J. Wickham ${ }^{7}$ Istvan Molnar ${ }^{7}$ and Denise A. Yardley ${ }^{8}$

\begin{abstract}
Background: Human epidermal growth factor receptor 2 (HER2)-positive breast cancer is a particularly aggressive form of the disease, and ultimately progresses in patients with metastases on standard therapies. Anthracyclines, such as doxorubicin, are an effective treatment for HER2-positive breast cancer, particularly when administered in combination with trastuzumab - however, doxorubicin-related cardiotoxicity has limited its use. Many patients are therefore never treated with anthracyclines, even upon disease progression, despite the potential for benefit. MM-302 is a novel, HER2-targeted antibody-liposomal doxorubicin conjugate that specifically targets HER2overexpressing cells. Preclinical and Phase 1 data suggest that MM-302, as a monotherapy or in combination with trastuzumab, could be effective for managing previously treated, anthracycline-naïve, HER2-positive breast cancer, without the cardiotoxicity observed with free doxorubicin formulations.

(Continued on next page)
\end{abstract}

\footnotetext{
*Correspondence: jreynolds@merrimackpharma.com

Previous presentation: Miller K et al. San Antonio Breast Cancer Symposium (SABCS) 2015, Poster OT3-01-01

${ }^{7}$ Merrimack Pharmaceuticals, Inc., 1 Kendall Square, Suite B7201, Cambridge,

MA 02139-1670, USA

Full list of author information is available at the end of the article
} 
(Continued from previous page)

Methods/Design: HERMIONE is an open-label, multicenter, randomized (1:1) Phase 2 trial of MM-302 plus trastuzumab versus chemotherapy of physician's choice (gemcitabine, capecitabine, or vinorelbine) plus trastuzumab planned to enroll 250 anthracycline-naïve patients with locally advanced/metastatic HER2-positive breast cancer. Key inclusion criteria are: previous treatment with trastuzumab (with or without pertuzumab) in any setting; refractory or intolerant to pertuzumab (refractory to pertuzumab defined as progression in the locally advanced or metastatic setting, or disease recurrence during or within 12 months of completing pertuzumab-containing neoadjuvant and/or adjuvant therapy); and disease progression on, or intolerant to, ado-trastuzumab emtansine for locally advanced or metastatic disease. The trial is currently being conducted at sites in the USA, Canada, and Western Europe. Treatment will be administered in 21-day cycles, and will be continued until disease progression or unacceptable toxicity. The primary endpoint is independently assessed progression-free survival (PFS). Tumor response will be assessed every 6 weeks, and defined according to RECIST v1.1. Secondary endpoints include investigator-assessed PFS, overall survival (OS), OS rates at 6 months and 1 year, objective response rates, safety and tolerability, quality of life, and the pharmacokinetic profile of MM-302 plus trastuzumab.

Discussion: The HERMIONE study will evaluate the efficacy and safety of MM-302 plus trastuzumab in patients with refractory HER2-positive advanced/metastatic breast cancer for whom there are no standard of care therapies with a proven survival advantage.

Trial Registration: Clinicaltrials.gov identifier: NCT02213744. Registration date: 06AUG2014.

Keywords: Advanced/metastatic breast cancer, Antibody-conjugate, Doxorubicin, Cardiotoxicity, HERMIONE, Human epidermal growth factor receptor 2/HER2/Erb2, HER2-targeted liposomal doxorubicin, Immunoliposome, MM302, Trastuzumab

\section{Background}

Approximately $20 \%$ of breast cancers are human epidermal growth factor receptor 2 (HER2)-positive, which represents a particularly aggressive subtype $[1,2]$. HER2-targeted anticancer therapies such as trastuzumab, pertuzumab, ado-trastuzumab emtansine (T-DM1), and lapatinib have transformed the management and prognosis of HER2-positive advanced/metastatic breast cancer [3-10]. Current standard of care is to continue HER2targeted therapy indefinitely (trastuzumab with changing chemotherapy partners, or T-DM1 or lapatinib in combination with capecitabine) [11-15]. Nonetheless, disease ultimately progresses in patients on standard therapies. Evidence suggests that limited mechanistic diversity associated with repeated use of microtubule-targeting chemotherapeutic agents, including taxanes (paclitaxel, nab-paclitaxel, and docetaxel) and vinca domainbinding agents (T-DM1, vinorelbine, and eribulin), could contribute to their diminishing effectiveness, owing to shared mechanisms of resistance [16]. Novel treatment strategies are essential.

\section{Advantages and limitations of existing anthracycline formulations}

Anthracyclines such as doxorubicin are effective for managing HER2-positive breast cancer, particularly in anthracycline-naïve patients. The trastuzumab registration study demonstrated increased efficacy with trastuzumab plus an anthracycline-containing regimen compared with an anthracycline-containing regimen alone in advanced/metastatic breast cancer [17]. However, this study also reported substantial cardiotoxicity when combining an anthracycline with trastuzumab: cardiac dysfunction occurred in $27 \%$ of patients who received trastuzumab plus an anthracycline-based regimen (with severe heart failure reported in $16 \%$ of patients), compared with $8 \%$ for an anthracyclinebased regimen without trastuzumab, and $13 \%$ for paclitaxel plus trastuzumab. Furthermore, studies in the adjuvant and neoadjuvant settings suggest equivalent or greater efficacy of taxane-based regimens relative to anthracycline-based regimens in breast cancer $[18,19]$. These findings have led to a decline in anthracycline use overall (Fig. 1), and particularly in the adjuvant setting in HER2-positive advanced/metastatic breast cancer [18, 20-24].

PEGylated (PLD; Doxil ${ }^{\circ} /$ Caelyx $^{\circ}$, Janssen) and nonPEGylated (NPLD; Myocet $^{\circ}$, Enzon Pharmaceuticals) liposomal doxorubicin formulations have been developed that aim to overcome the challenges associated with using free doxorubicin. Both PLD and NPLD have shown reduced cardiotoxicity compared with free doxorubicin [25-32]. Although PLD appears to be active in this setting, PLD as a monotherapy or in combination with trastuzumab is not considered a standard of care in the treatment of HER2-positive advanced/metastatic 


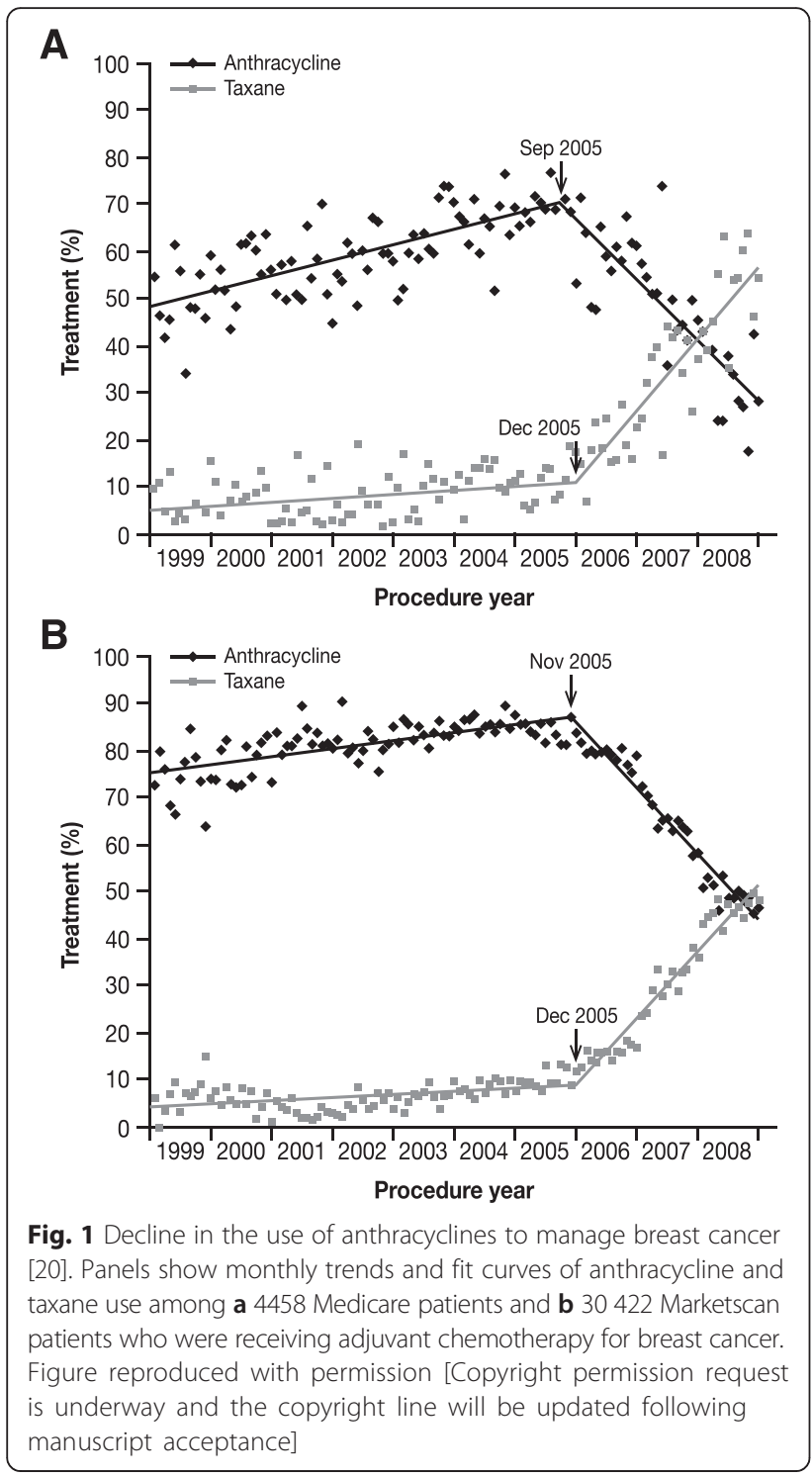

breast cancer. In Europe, PLD monotherapy is approved for metastatic breast cancer patients at high cardiac risk [33], but in the USA, PLD has not been approved either alone or in combination with trastuzumab. NPLD in combination with trastuzumab and paclitaxel was not superior to the combination of trastuzumab and paclitaxel in a Phase 3 study of first-line therapy for advanced/metastatic breast cancer [32].

\section{MM-302 - a novel, HER2-targeted antibody-liposomal doxorubicin conjugate}

MM-302 is a novel, HER2-targeted antibody-liposomal doxorubicin conjugate (Fig. 2) that specifically targets HER2overexpressing cells, increasing delivery of doxorubicin to tumor cells and limiting exposure to healthy cells such as cardiomyocytes (Fig. 3). MM-302 is therefore designed potentially to be better tolerated than free doxorubicin, and to be more effective than free doxorubicin and existing liposomal formulations in HER2overexpressing tumors [34, 35]. In preclinical models, MM-302 demonstrated superior antitumor activity compared with both free doxorubicin and PLD [35]. Moreover, MM302 and trastuzumab bind to different HER2 epitopes, and the combination of MM-302 and trastuzumab demonstrated superior antitumor activity to either agent alone in HER2overexpressing tumor xenograft models (unpublished observations, Chris Espelin, Shannon Leonard, and Elena Geretti; Merrimack Pharmaceuticals, Inc., Cambridge, MA, USA). These findings provided support for investigating the combination in the clinical setting. Phase 1 data suggest that MM-302 alone or in combination with trastuzumab has promising efficacy and a manageable safety profile in patients with advanced HER2-positive breast cancer. In the population treated with $\geq 30 \mathrm{mg} / \mathrm{m}^{2} \mathrm{MM}-302$ alone $(n=34)$ or combined with either trastuzumab $(n=22)$ or trastuzumab plus cyclophosphamide $(n=13)$, the objective response rate was $11.3 \%(n=7 / 62)$ and median progression-free survival (PFS) was 7.6 months (95\% CI: 3.6-10.9). Increased efficacy was observed in the anthracyclinenaïve subgroup, with an objective response rate of $24.0 \%(n=6 / 25)$ and median PFS of 11.0 months (95 \% CI: 1.8-13.1) [36]. No cardiac adverse events were reported with MM-302 monotherapy; in the combination arms, cardiac adverse events were infrequent and none were serious adverse events [36].

\section{Rationale for the HERMIONE trial}

Given the high unmet need for novel treatment options for patients with HER2- positive metastatic breast cancer that has progressed on pertuzumab, trastuzumab, and T-DM1, and the promising activity seen in the Phase 1 study, the Phase 2 HERMIONE trial has been designed, with input from the US Food and Drug Administration (FDA) and review by the European Medicines Agency (EMA), to assess the efficacy and tolerability of MM-302 in combination with trastuzumab, compared with chemotherapy of physician's choice plus trastuzumab, in patients with previously treated, anthracycline-naïve, HER2positive locally advanced/metastatic breast cancer.

\section{Methods/Design}

Study design

HERMIONE is an open-label, multicenter, randomized (1:1) Phase 2 trial of MM-302 plus trastuzumab versus chemotherapy of physician's choice (gemcitabine, capecitabine, or vinorelbine) plus trastuzumab in anthracycline-naïve patients with locally advanced/ metastatic HER2-positive breast cancer (ClinicalTrials.gov Identifier: NCT02213744). The trial is being 


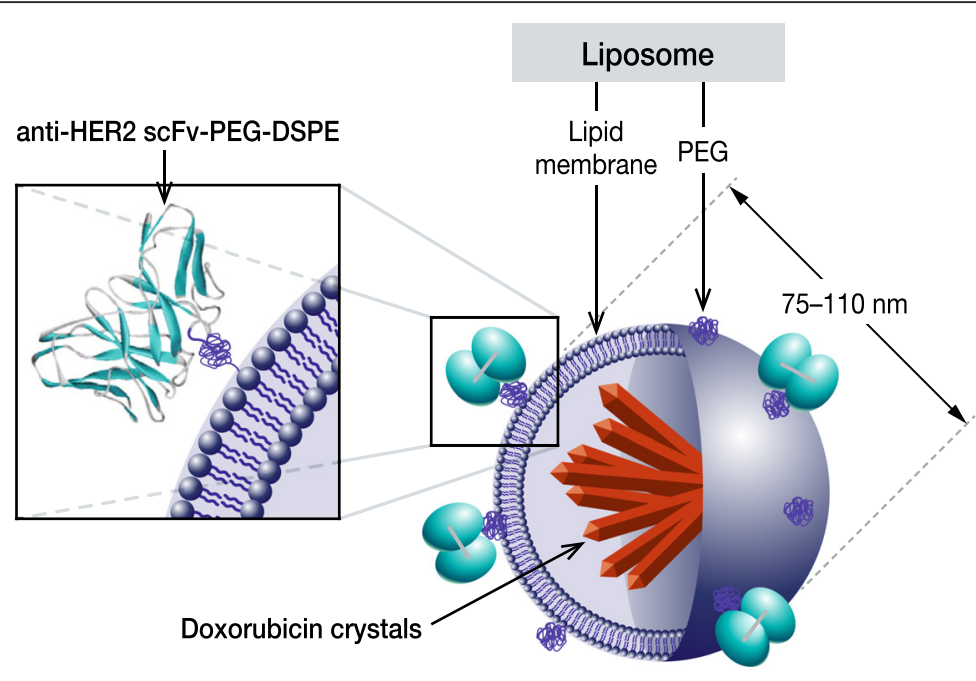

DSPE: distearoylphosphatidylethanolamine;

HER2: human epidermal growth factor receptor 2; PEG: polyethylene glycol; scFv: single chain fraction variable.

Fig. 2 Schematic of MM-302, a novel HER2-targeted antibody-liposomal doxorubicin conjugate. MM-302 consists of doxorubicin encapsulated by a liposome that is conjugated to an anti-HER2 scFv antibody via a polyethylene glycol spacer (PEG-DSPE). MM-302 thus directly targets PEGylated liposomal doxorubicin to HER2-overexpressing tumor cells

conducted at sites in the USA, Canada, and Western Europe. The trial design and treatment schedule are summarized in Fig. 4.

Patients will be randomized according to a prespecified randomization scheme generated by an independent statistician. Once patient eligibility for the study has been confirmed, investigational sites will log into a computerized interactive web response system to obtain treatment arm assignment. Randomization will be stratified based on world region (North America/Western Europe/Other), presence of visceral disease (yes/no), and number of prior lines of anticancer therapy for locally advanced/metastatic disease $(\leq 3 / \geq 4)$.

The study will be performed according to the principles of the Declaration of Helsinki, the International Conference on Harmonization Guidance on Good Clinical Practice, and the requirements of the US FDA and/or local regulatory authorities regarding the conduct of human clinical trials. All patients must give written informed consent. Local Institutional Review Board or Ethics Committee approval of the protocol, informed consent document, and any other material used to inform the patient about the nature of the trial has been obtained for all participating centers.

\section{Eligibility criteria}

Key inclusion criteria are: histologically or cytologically confirmed invasive breast cancer that is centrally confirmed as HER2-positive by American Society of Clinical Oncology/College of American Pathologists
2013 guidelines [37]; documented locally advanced/ metastatic disease; prior treatment with trastuzumab in any setting; refractory or intolerant to pertuzumab (refractory to pertuzumab is defined as progression on pertuzumab in the locally advanced or metastatic setting, or development of disease recurrence during or within 12 months of completing pertuzumab-containing neoadjuvant and/or adjuvant therapy); disease progression on, or intolerant to, T-DM1 treatment for locally advanced or metastatic disease; not previously treated with an anthracycline in any setting; aged $\geq 18$ years; Eastern Cooperative Oncology Group performance status 0 or 1; left ventricular ejection fraction (LVEF) $\geq 50 \%$. Patients with treated, stable, asymptomatic central nervous system (CNS) metastases and who have not received steroid treatment for 4 weeks prior to enrollment may be considered for this trial. Further details of the study inclusion and exclusion criteria are presented in Table 1 , and examples of the most common previous treatment pathways for eligible patients are shown in Fig. 5. There is no restriction on the number of prior lines of therapy for eligible patients.

\section{Study treatments Treatment arms}

Patients will be randomized to receive either MM-302 plus trastuzumab or chemotherapy of physician's choice plus trastuzumab. In the experimental arm, patients will receive MM-302 $30 \mathrm{mg} / \mathrm{m}^{2}$ IV on day 1 of each 21-day cycle, and trastuzumab $8 \mathrm{mg} / \mathrm{kg}$ IV (loading dose) and 

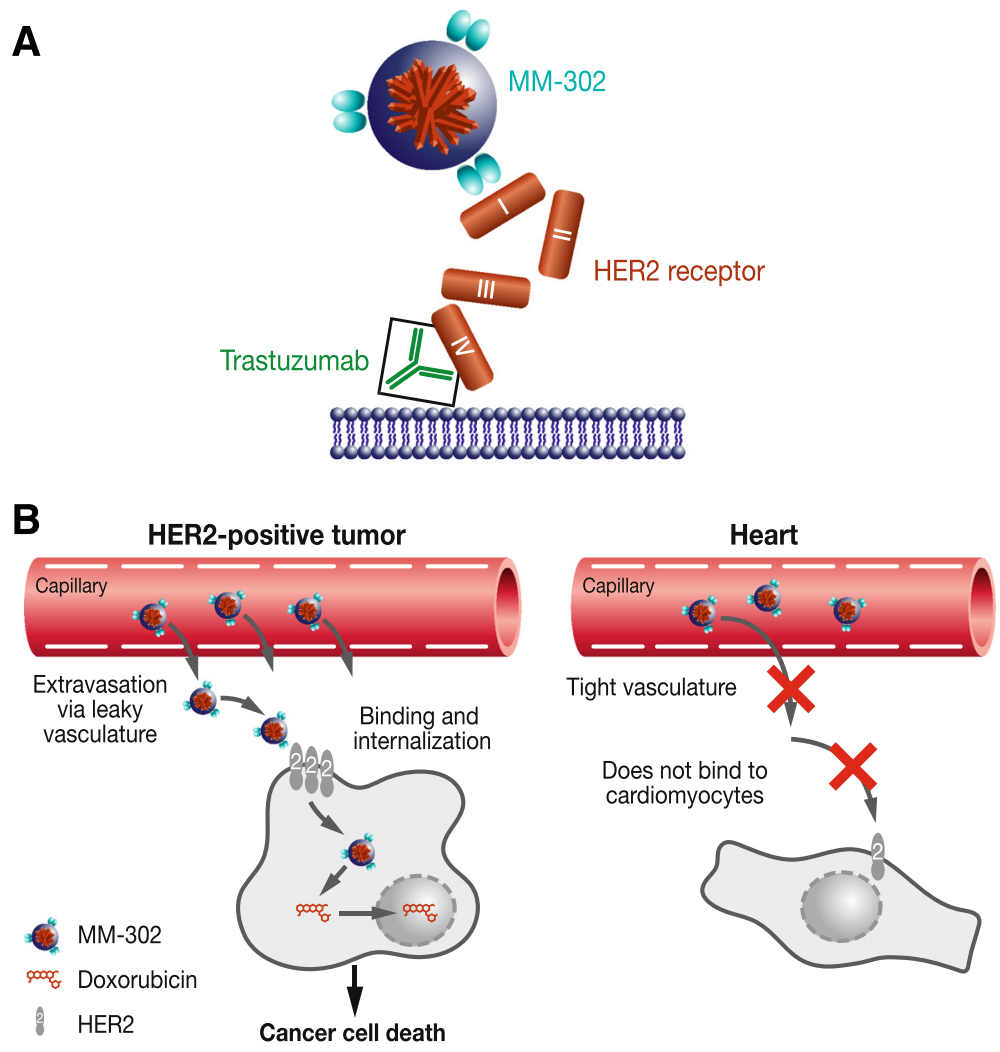

HER2: human epidermal growth factor receptor 2.

Fig. 3 Mechanism of action of MM-302. a MM-302 binds to HER2 extracellular subdomain I, whilst trastuzumab binds to subdomain IV. b MM-302 remains in circulation for long periods of time, providing an opportunity to accumulate in tumors via leaky vasculature. Once in the tumor microenvironment, MM-302 binds specifically to tumor cells that overexpress HER2 (>200 000/cell) and undergoes receptor-mediated endocytosis, releasing doxorubicin inside the cell. By contrast, the vasculature of the heart is more intact and prevents extravasation out of the blood vessels. Furthermore, cardiomyocytes express HER2 below the threshold required for uptake; therefore, MM-302 does not inhibit HER2-mediated signaling in cardiomyocytes $[34,35]$

$6 \mathrm{mg} / \mathrm{kg}$ IV (maintenance dose) on day 1 of each 21-day cycle. In the control arm, physicians will select a chemotherapy (limited to gemcitabine, capecitabine, or vinorelbine) plus trastuzumab, as follows: gemcitabine $1000-1250 \mathrm{mg} / \mathrm{m}^{2}$ IV on days 1 and 8 of each 21 -day cycle; capecitabine $1000-1250 \mathrm{mg} / \mathrm{m}^{2}$ twice daily, administered orally on days 1-14 of each 21-day cycle; vinorelbine $25-30 \mathrm{mg} / \mathrm{m}^{2}$ IV on days 1 and 8 (and optionally on day 15) of each 21-day cycle; trastuzumab administration in the control arm is the same as in the experimental arm. Treatment will be continued until progression or intolerable toxicity. There will be no crossover of control arm to receive study drug on progression.

\section{Dose modifications}

Dose modification of study treatments is permitted to manage toxicities. A maximum of two MM-302 dose reductions (by $25 \%$ ) are permitted to manage hematologic and non-hematologic adverse events. For hepatotoxicity, the dose will be reduced to $15 \mathrm{mg} / \mathrm{m}^{2}$ if total bilirubin is $1.2-3.0 \mathrm{mg} / \mathrm{dL}$, and to $7.5 \mathrm{mg} / \mathrm{m}^{2}$ if total bilirubin is $>3.0 \mathrm{mg} / \mathrm{dL}$. Any patients requiring a third dose reduction will have MM-302 discontinued. Specific MM-302 dose modification criteria are also defined for managing changes in LVEF. In case of persistent asymptomatic LVEF decreases and congestive heart failure, study treatment will be permanently discontinued. Patients with confirmed symptoms of congestive heart failure will also discontinue treatment permanently.

Specific criteria to withhold/discontinue MM-302 treatment are also defined for managing LVEF changes. MM-302 will be withheld if LVEF declines to $\leq 45 \%$ or if LVEF declines to $46-49 \%$ and is $\geq 15 \%$ points below baseline. LVEF assessment will then be repeated after 3 weeks: if LVEF recovers sufficiently (LVEF $\geq 50 \%$, or $46-49 \%$ and $<15 \%$ points below baseline), study 


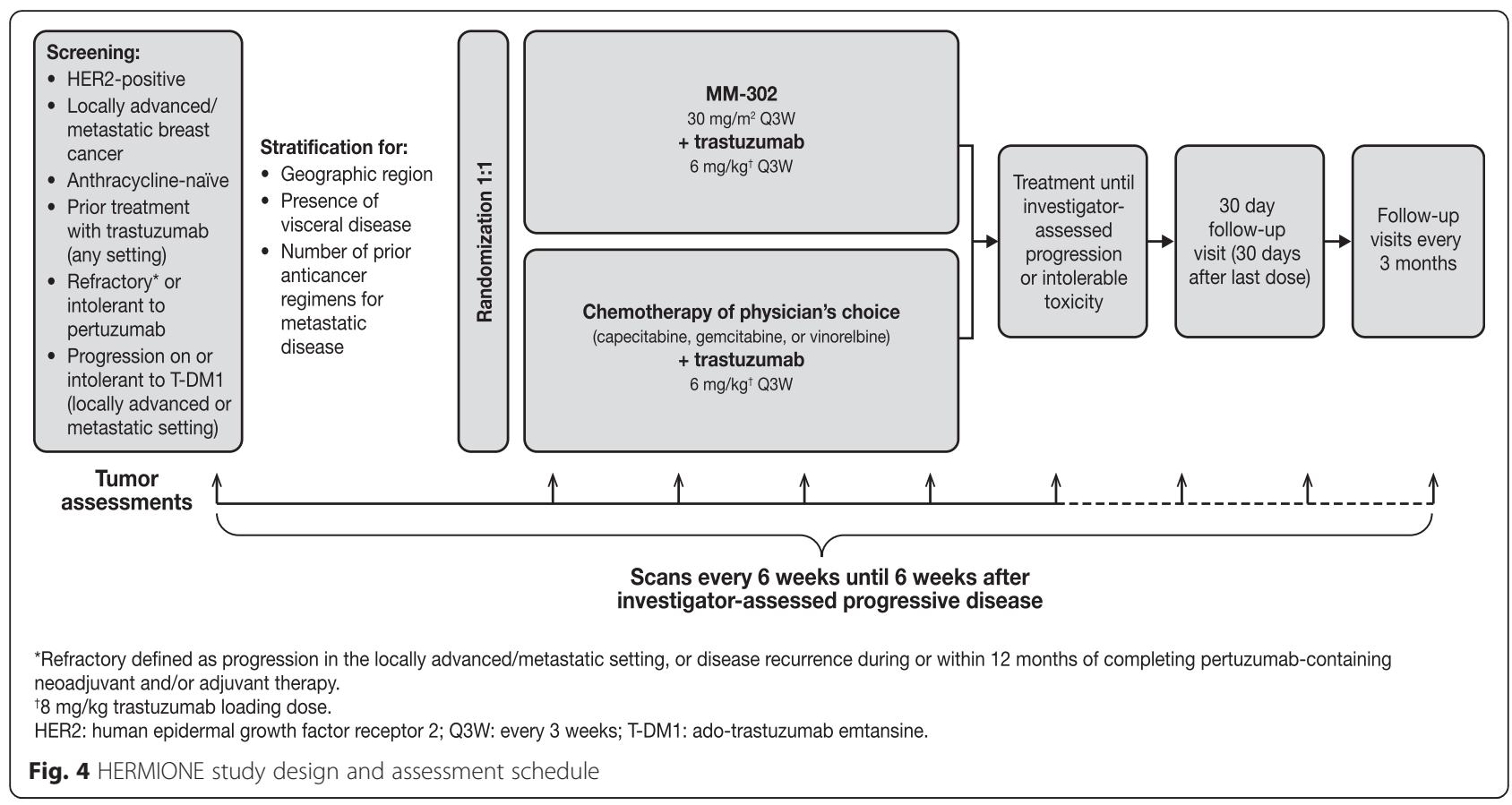

treatment will be resumed, otherwise the patient will permanently discontinue treatment.

\section{Concomitant therapies}

Patients may receive concomitant analgesics, antiemetics, antidiarrheal antibiotics, antipyretics, hematopoietic growth factors, and blood products as per physician discretion. For patients with bone metastases, standard of care treatments such as bisphosphonates and denosumab are permitted. The following therapies are not permitted while on study treatment: other antineoplastic therapies; radiotherapy (unless given for palliative reasons and with the approval of the Medical Monitor); systemic prophylactic corticosteroids prior to first administration of MM-302; any other investigational therapies; and any therapies that are prohibited from being given concomitantly with gemcitabine, capecitabine, or vinorelbine, as described in the respective national prescribing information.

\section{Study objectives}

The primary objective is to determine whether the combination of MM-302 plus trastuzumab improves PFS (time from randomization until documented disease progression or death) compared with chemotherapy of physician's choice plus trastuzumab. PFS will be assessed by a blinded independent review. Secondary endpoints are investigator-assessed PFS; overall survival (OS; time from randomization until death); OS rate at 6 months and 1 year; time to treatment failure (time from randomization until treatment discontinuation for any reason, including disease progression, treatment toxicity, or death); objective response rate (patients with confirmed complete or partial response); duration of response (time from first, documented, confirmed objective response until objectively documented recurrent or progressive disease); safety profiles; and pharmacokinetic exposure of MM-302 and trastuzumab. Exploratory objectives include the correlation between biomarkers and clinical outcome; time to symptomatic progression; patient-reported outcomes (Functional Assessment of Cancer Therapy-Breast [FACT-B] and EuroQol [EQ]-5D); population pharmacokinetics of MM-302 including estimations of interpatient variability of pharmacokinetic parameters; rate and time to development of CNS progression and new CNS metastases; estimation of resources for hospitalizations/hospital visits during the study and within 30 days of the last dose of study treatment; and concordance between independently and investigator-assessed PFS. An independent Cardiac Review Committee will monitor cardiac safety, and a Data Safety Monitoring Board will monitor the overall safety of the study.

\section{Study assessments}

Tumor response will be assessed by computed tomography/magnetic resonance imaging at screening and every 6 weeks from randomization, and will be defined according to Response Evaluation Criteria In Solid Tumors (RECIST) v1.1. Cardiotoxicity will be assessed by monitoring LVEF at screening and every 6 weeks from randomization until 6 weeks after 
Table 1 Key inclusion and exclusion criteria for the Phase 2 HERMIONE study

\begin{tabular}{|c|c|}
\hline Criteria & Details \\
\hline \multicolumn{2}{|l|}{ Inclusion criteria } \\
\hline Disease-specific & $\begin{array}{l}\text { - Histologically or cytologically confirmed invasive cancer of the breast, with documented locally } \\
\text { advanced/metastatic disease that is not amenable to resection with curative intent. Cancer must } \\
\text { be HER2-positive, as defined by ASCO/CAP } 2013 \text { guidelines [37]. } \\
\text { - Documented disease progression (via RECIST or clinical progression) or intolerance during or } \\
\text { after the most recent treatment for locally advanced/metastatic breast cancer. } \\
\text { - Refractory or intolerant to pertuzumab (refractory to pertuzumab is defined as progression on } \\
\text { pertuzumab in the locally advanced or metastatic setting, or development of disease recurrence } \\
\text { during or within } 12 \text { months of completing pertuzumab-containing neoadjuvant and/or adjuvant } \\
\text { therapy). } \\
\text { - Disease progression on, or intolerant to, T-DM1 in the locally advanced/metastatic breast } \\
\text { cancer setting. } \\
\text { - Previously treated with trastuzumab in any setting (trastuzumab could have been previously } \\
\text { administered with or without pertuzumab). }\end{array}$ \\
\hline General & $\begin{array}{l}\text { - Age } \geq 18 \text { years. } \\
\text { - Eastern Cooperative Oncology Group performance status } 0 \text { or } 1 .\end{array}$ \\
\hline $\begin{array}{l}\text { Hematologic, biochemical, and organ } \\
\text { function }\end{array}$ & $\begin{array}{l}\text { - Eligible to receive at least one of gemcitabine, capecitabine, or vinorelbine. } \\
\text { - Adequate bone marrow reserves (absolute neutrophil count } \geq 1500 / \mu \mathrm{L} ; \text { platelet count } \geq 100000 / \mu \mathrm{LL} \text {; } \\
\text { hemoglobin } \geq 9 \mathrm{~g} / \mathrm{dL} \text { [transfusions allowed]), coagulation function (INR and aPTT } \leq 1.5 \cup L N \text {, unless } \\
\text { on therapeutic coagulants), hepatic function (serum total bilirubin within normal limits; AST and ALT } \\
\text { up to } 3 x \text { ULN; serum albumin } \geq 2.5 \mathrm{~g} / \mathrm{dL} \text { ), renal function (serum creatinine } \leq 1.5 \times U L N \text { ), and cardiac } \\
\text { function (LVEF } \geq 50 \% \text { by MUGA or ECHO). }\end{array}$ \\
\hline \multicolumn{2}{|l|}{ Exclusion criteria } \\
\hline Disease-specific & $\begin{array}{l}\text { - Previous treatment with doxorubicin, liposomal doxorubicin, epirubicin, mitoxantrone, or any other } \\
\text { anthracycline derivative. } \\
\text { - CNS metastases, unless patients have been treated and are stable without symptoms for } 4 \text { weeks } \\
\text { after completion of treatment, and they must be off steroids for at least } 4 \text { weeks prior to enrollment } \\
\text { - Active other malignancy or history of other malignancy within the last } 5 \text { years except appropriately } \\
\text { treated carcinoma of the cervix, non-melanoma skin carcinoma, stage } 1 \text { uterine cancer, or cancers } \\
\text { with a similar curative outcome as those previously mentioned. } \\
\text { - Known hypersensitivity to any of the components of MM-302 or hypersensitivity reactions to fully } \\
\text { humanized monoclonal antibodies. } \\
\text { - History of intolerance to trastuzumab. Patients who have been successfully re-challenged with } \\
\text { trastuzumab after a mild infusion reaction are allowed. } \\
\text { - Investigational therapy administered }<28 \text { days (or }<5 \text { half-lives; whichever is the longest) prior to } \\
\text { the first scheduled day of study drug dosing. } \\
\text { - Any standard anticancer therapy }<14 \text { days prior to the first scheduled day of study drug dosing } \\
\text { (except trastuzumab). }\end{array}$ \\
\hline Cardiac & $\begin{array}{l}\text { - Any class of NYHA congestive heart failure, or heart failure with preserved ejection fraction. } \\
\text { - History of known coronary artery disease or a myocardial infarction within the last } 12 \text { months. } \\
\text { - Uncontrolled hypertension (SBP }>160 \mathrm{mmHg} \text { or DBP }>100 \mathrm{mmHg} \text { ). } \\
\text { - Unstable angina pectoris. } \\
\text { and paroxysmal supraventricular tachycardia). } \\
\text { - Prolonged QTc interval ( } \geq 450 \mathrm{~ms} \text { ). } \\
\text { - Previous discontinuation of trastuzumab due to unacceptable cardiac toxicity or infusion-related } \\
\text { reactions. } \\
\text { - History of LVEF decline to }<50 \% \text { during or after HER2-directed therapy. } \\
\text { - Current dyspnea at rest that requires continuous oxygen therapy. }\end{array}$ \\
\hline General & $\begin{array}{l}\text { - Pregnant or breast feeding. } \\
\text { - Active infection or unexplained fever }>38.5^{\circ} \mathrm{C} \text { during screening visits. } \\
\text { - History of allogeneic transplant (patients with a history of autologous bone marrow or stem } \\
\text { cell transplant may be enrolled). }\end{array}$ \\
\hline
\end{tabular}

ALT alanine aminotransferase, aPTT activated partial thromboplastin time, ASCO American Society of Clinical Oncology, AST aspartate aminotransferase, CAP College of American Pathologists, CNS central nervous system, CTCAE Common Terminology Criteria for Adverse Events, DBP diastolic blood pressure, ECHO echocardiogram, HER2 human epidermal growth factor receptor 2, INR international normalized ratio, LVEF left ventricular ejection fraction, MUGA multiple-gated acquisition scan, NYHA New York Heart Association, QTC corrected QT interval, RECIST Response Evaluation Criteria In Solid Tumors, SBP systolic blood pressure, $T$-DM1 ado-trastuzumab emtansine, ULN upper limit of normal

treatment discontinuation. LVEF will be determined by multiple-gated acquisition scan or echocardiogram, with the same assessment method continued throughout the study. Long-term cardiotoxicity will be assessed by recording the occurrence of symptomatic heart failure in the follow-up period of the study. Safety will be assessed 


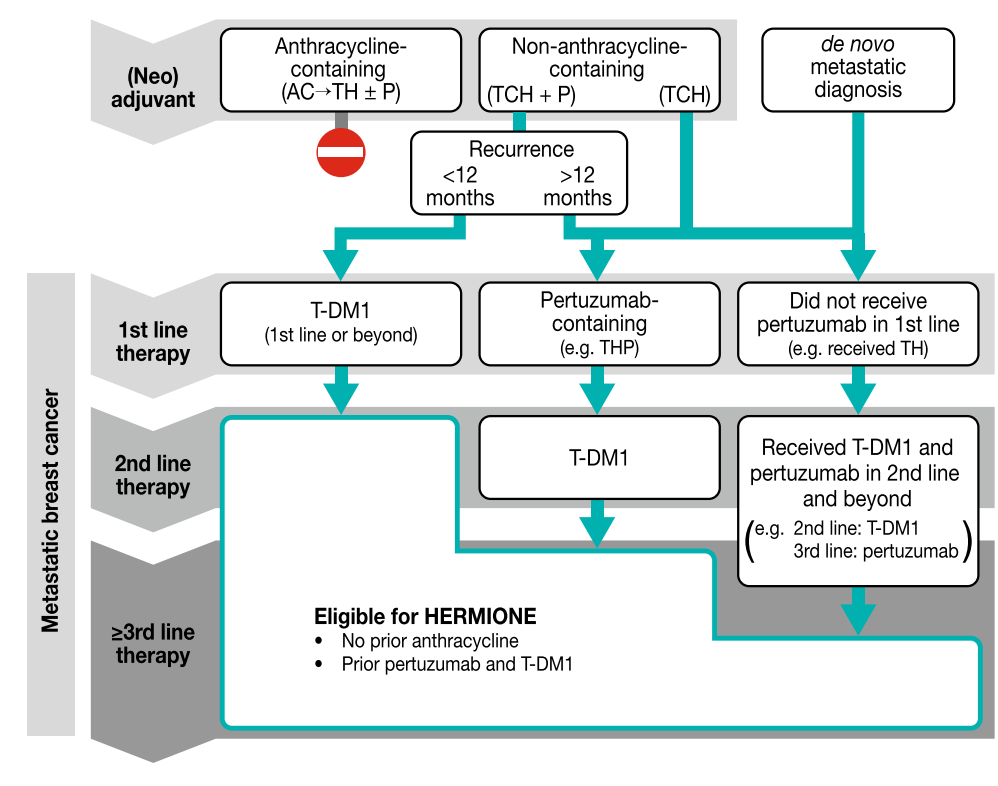

AC: anthracycline; P: pertuzumab; T-DM1: ado-trastuzumab emtansine; TCH: taxane + carboplatin + trastuzumab; TH: taxane + trastuzumab; THP: taxane + trastuzumab + pertuzumab.

Fig. 5 Examples of the most common previous treatment pathways for eligible patients

using the National Cancer Institute Common Terminology Criteria for Adverse Events (NCI-CTCAE) v4.0 at screening; on days 1,8 , and 15 of cycle 1 ; on day 1 of every cycle thereafter; and 30 days after treatment discontinuation. Blood samples for pharmacokinetic analyses will be taken in cycles 1 and 2 from patients randomized to MM302 and trastuzumab; samples will be collected preinfusion, immediately post-infusion, 8-96 h post-infusion (optional sample), and $168 \mathrm{~h}$ post-infusion. Patient quality of life will be assessed using the FACT-B and EQ-5D instruments. For analysis of serum biomarkers (in patients who consent) and anti-MM-302 immunogenicity (in the MM-302 arm only), serum blood samples will be collected before dosing on day 1 of cycle 1 and every cycle thereafter and 30 days after treatment discontinuation.

\section{Statistical analysis}

The planned sample size is approximately 250 patients (125 per arm). This sample size was chosen to observe 191 events, providing at least $90 \%$ power to detect an approximately $60 \%$ improvement in PFS (hazard ratio of 0.625 ). The study powering assumes a median PFS of 8 months and 5 months in the experimental arm and control arm, respectively.

Efficacy will be assessed in the intent-to-treat population, which will include all randomized patients. Timeto-event analyses, including the primary endpoint, will be compared between the experimental and control arms using a stratified log-rank test at a 2 sided $5 \%$ significance level in the intent-to-treat population. Timeto-event curves will be estimated using Kaplan-Meier estimates. Hazard ratios and $95 \%$ confidence intervals will be estimated using Cox proportional hazard models. Objective response rate will be assessed using a stratified Mantel-Haenszel test. The safety population will include all patients who receive at least one dose (including a partial dose) of study medication; safety will be assessed using descriptive statistics.

\section{Discussion}

The Phase 2 HERMIONE trial has been designed, with input from the US FDA and review by the EMA, to assess the efficacy and tolerability of MM302 in combination with trastuzumab, compared with chemotherapy of physician's choice plus trastuzumab, in anthracycline-naive patients with HER2positive locally advanced/metastatic breast cancer previously treated with pertuzumab or T-DM1. Recruitment to the trial began in September 2014.

HERMIONE is investigating a patient population with a high unmet need for effective therapies. The standard of care for advanced/metastatic HER2-positive breast cancer is to continue HER2-targeted therapy indefinitely (trastuzumab with changing chemotherapy partners, or T-DM1 or lapatinib in combination with capecitabine) [11-15]. Microtubule-targeting chemotherapies, including taxanes (paclitaxel, nab-paclitaxel, and docetaxel) and vinca domain-binding agents (T-DM1, vinorelbine, and eribulin), are frequently used. However, common resistance mechanisms are likely to occur among microtubule-targeted agents and especially within the specific classes [16]. These shared resistance mechanisms 
could result in diminishing treatment benefit for patients. In addition, successive use of microtubule-targeting agents may pose increasing safety concerns due to cumulative neuropathy. Apart from the microtubule-targeting agents, the most frequently used chemotherapeutics in combination with trastuzumab include capecitabine, and gemcitabine $[8,15]$. Many patients continue to have a good performance status after disease progression on standard HER2-targeted therapies and continue to be candidates for additional treatment. Given the heterogeneity of advanced breast cancer, a mechanistically diverse range of therapeutic options is warranted.

The study was designed to recruit anthracycline-naïve patients following preliminary efficacy data suggesting that treatment with MM-302 results in higher response rates and longer median PFS in anthracycline-naïve patients, compared with those previously exposed to anthracyclines [36, 38], consistent with published literature on PLD [26, 39, 40]. In addition, the safety concerns over the use of anthracyclines in combination with trastuzumab have led to a decline in anthracycline use, resulting in a large pool of patients who are anthracycline-naïve, and who have not received one of the most active classes of chemotherapy for the treatment of breast cancer [20]. The development of novel formulations of these agents may therefore extend the benefits of this class to this group of patients.

Patients enrolling in HERMIONE who have already been exposed to trastuzumab will continue trastuzumab treatment. As noted earlier, this is in line with the current standard of care for HER2-positive breast cancer of continuing HER2-targeted therapy indefinitely [11-15]. Supporting this practice, a separate clinical study randomized patients with HER2-positive breast cancer whose disease had progressed on trastuzumab treatment to receive capecitabine alone or capecitabine plus trastuzumab. The study results showed a significant improvement in overall response and PFS with capecitabine plus trastuzumab compared with capecitabine alone [12, 13], supporting the continued use of trastuzumab plus chemotherapy in HER2-positive breast cancer.

The MM-302 dose of $30 \mathrm{mg} / \mathrm{m}^{2}$ every 21 days was selected based on safety and efficacy data from an ongoing Phase 1 trial: MM-302 was well tolerated at doses of $30-40 \mathrm{mg} / \mathrm{m}^{2}$ but an increase in grade $3 / 4$ adverse events was observed at $50 \mathrm{mg} / \mathrm{m}^{2}$ administered every 4 weeks (the highest dose level evaluated) $[36,38]$. The 21-day cycle for MM-302 was selected to simplify combination with the approved trastuzumab regimen, as this is also administered every 21 days [38].

The control arm will receive trastuzumab added to chemotherapy of physician's choice, either gemcitabine, capecitabine, or vinorelbine. These are three of the most commonly used cytotoxic agents (besides taxanes, which will have been used in earlier lines of therapy); they can be combined with trastuzumab [8, 12, 13, 41-44]; and they are recommended in treatment guidelines [11, 14, 15]. Chemotherapy of physician's choice is limited to only three agents to minimize potential confounding of the key outcome measures, given the relatively small sample size required for this study. There is flexibility in the dose ranges that physicians can use, in line with the approved US package insert or European Summary of Product Characteristics, and the National Comprehensive Cancer network guidelines $[15,45-50]$, reflecting doses commonly used in the clinic.

The primary endpoint for the HERMIONE trial is PFS as determined by a blinded independent review, based on evidence that increases in PFS have generally translated into improvements in OS in late-line HER2positive advanced/metastatic breast cancer. An analysis of approval trials in HER2-positive advanced/metastatic breast cancer suggested a moderate correlation between PFS and OS [51]. Furthermore, the TH3RESA trial reported a PFS hazard ratio of 0.53 that corresponded with an OS hazard ratio of 0.55 from the first interim analysis [8]. This trial evaluated T-DM1 in HER2-positive lateline advanced/metastatic breast cancer, and used a comparator arm of treatment of physician's choice, which was predominantly trastuzumab in combination with chemotherapy. These data suggest that an improvement in PFS with trastuzumab in combination with MM-302 in a similar patient population to that in TH3RESA could likewise translate into an improvement in OS.

\section{Conclusion}

The HERMIONE trial is designed to test whether MM-302, a novel HER2-targeted antibody-liposomal doxorubicin conjugate, added to trastuzumab could be an effective and welltolerated treatment option for anthracycline-naïve patients with HER2-positive advanced/metastatic breast cancer who are candidates for systemic therapy after disease progression on approved agents.

\section{Additional file}

\section{Additional file 1: HERMIONE IRB Data. (XLSX $15 \mathrm{~kb}$ )}

\section{Abbreviations}

CNS, central nervous system; EMA, European Medicines Agency; EQ, EuroQol; FACT-B, functional assessment of cancer therapy-breast; FDA, Federal Drug Administration; HER2, human epidermal growth factor receptor 2; LVEF, left ventricular ejection fraction; NCI-CTCAE, National Cancer Institute Common Terminology Criteria for Adverse Events; NPLD, non-PEGylated liposomal doxorubicin; OS, overall survival; PFS, progression-free survival; PLD, pEGylated liposomal doxorubicin; RECIST, response evaluation criteria in solid tumors; T-DM1, Ado-trastuzumab emtansine; VHIO, Vall d'Hebron Institute of Oncology. 


\section{Acknowledgements}

We thank all the patients, caregivers, and investigators who are participating in this study. We also thank Emma Robinson from 7.4 Limited, who provided medical writing support funded by Merrimack Pharmaceuticals. The HERMIONE trial is sponsored by Merrimack Pharmaceuticals. The study design has been funded by Merrimack Pharmaceuticals, and the collection, analysis, and interpretation of data will be funded by Merrimack Pharmaceuticals. The sponsor provided a formal review of the manuscript but the authors had final authority, including final responsibility to submit for publication.

\section{Funding}

This study is commercially funded by Merrimack Pharmaceuticals.

\section{Availability of data and materials}

The trial is ongoing and no data is available.

\section{Authors' contributions}

Wrote and/or reviewed study protocol: KR, JC, JR. Obtained ethical approval: DT, IK, SV. Contributed to study design: DT, DY, IK, JC, JR, KM, KR, SH, SV, TW. Will provide patient care: DT, DY, IK, JC, SH, SV. Will collect study data: DT, DY, JC, SH, SV. Will provide medical supervision and trial monitoring: DT, DY, IM, JC, SV. Will analyze study data: DT, DY, IM, JC, JR, SV. All authors contributed to the writing and review of the manuscript and all read and approved the final manuscript.

\section{Authors' information}

No additional information provided.

\section{Competing interests}

DY has no competing interests.

KM and DT receive support for clinical trials (paid to institution) from Merrimack Pharmaceuticals. DT receives support for clinical trials (paid to the institution) from Novartis.

IK receives support for clinical trials (paid to institution) from Genentech/Roche. $\mathrm{JC}$ acts as a consultant for Roche and Celgene, and has received honoraria from Roche, Celgene, Eisai, and Novartis.

$\mathrm{SH}$ receives support for clinical trials (paid to institution) from Merrimack Pharmaceuticals, GlaxoSmithKline, and Genentech/Roche.

SV has acted as a consultant for Amgen, AstraZeneca, Eisai, Novartis, Pfizer, and Roche.

$\mathrm{KR}, J \mathrm{~J}, \mathrm{TW}$, and IM are employees of Merrimack Pharmaceuticals.

\section{Consent for publication}

Not applicable.

\section{Ethics approval and consent to participate}

All patients must give written informed consent to participate in the trial. Local Institutional Review Board or Ethics Committee approval of the protocol, informed consent document, and any other material used to inform the patient about the nature of the trial has been obtained for all participating centers. Please refer to Supplementary information (Additional file 1) for a list of each participating center and the respective Institutional Review Board that approved the trial.

\begin{abstract}
Author details
'Indiana University Melvin and Bren Simon Cancer Center, Indianapolis, IN, USA. ${ }^{2}$ Vall d'Hebron Institute of Oncology (VHIO), Barcelona, Spain and Ramony Cajal University Hospital, Madrid, Spain. ${ }^{3}$ University of California Los Angeles, Los Angeles, CA, USA. ${ }^{4}$ Dana-Farber Cancer Institute, Boston, MA, USA. ${ }^{5}$ MD Anderson Cancer Center, Houston, TX, USA. ${ }^{6}$ Sunnybrook Odette Cancer Centre, Toronto, Canada. ${ }^{7}$ Merrimack Pharmaceuticals, Inc., 1 Kendall Square, Suite B7201, Cambridge, MA 02139-1670, USA. ${ }^{8}$ Sarah Cannon Research Institute, and Tennessee Oncology, PLLC, Nashville, TN, USA.
\end{abstract}

\section{Received: 3 November 2015 Accepted: 25 May 2016}

Published online: 03 June 2016

\section{References}

1. Ross JS, Slodkowska EA, Symmans WF, Pusztai L, Ravdin PM, Hortobagyi GN. The HER-2 receptor and breast cancer: ten years of targeted anti-HER-2 therapy and personalized medicine. Oncologist. 2009;14:320-68.
2. Slamon DJ, Clark GM, Wong SG, Levin WJ, Ullrich A, McGuire WL. Human breast cancer: correlation of relapse and survival with amplification of the HER-2/neu oncogene. Science. 1987;235:177-82.

3. Baselga J, Cortes J, Kim SB, Im SA, Hegg R, Im YH, et al. Pertuzumab plus trastuzumab plus docetaxel for metastatic breast cancer. N Engl I Med. 2012;366:109-19.

4. Geyer CE, Forster J, Lindquist D, Chan S, Romieu CG, Pienkowski T, et al. Lapatinib plus capecitabine for HER2-positive advanced breast cancer. N Engl J Med. 2006;355:2733-43.

5. Verma S, Miles D, Gianni L, Krop IE, Welslau M, Baselga J, et al. Trastuzumab emtansine for HER2-positive advanced breast cancer. N Engl J Med. 2012;367:1783-91.

6. Swain SM, Baselga J, Kim SB, Ro J, Semiglazov V, Campone M, et al. Pertuzumab, trastuzumab, and docetaxel in HER2-positive metastatic breast cancer. N Engl J Med. 2015:372:724-34.

7. Wong DJ, Hurvitz SA. Recent advances in the development of anti-HER2 antibodies and antibody-drug conjugates. Ann Transl Med. 2014;2:122.

8. Krop IE, Kim SB, Gonzalez-Martin A, LoRusso PM, Ferrero JM, Smitt M, et al. Trastuzumab emtansine versus treatment of physician's choice for pretreated HER2-positive advanced breast cancer (TH3RESA): a randomised, open-label, phase 3 trial. Lancet Oncol. 2014;15:689-99.

9. Cameron D, Casey M, Oliva C, Newstat B, Imwalle B, Geyer CE. Lapatinib plus capecitabine in women with HER-2-positive advanced breast cancer: final survival analysis of a phase III randomized trial. Oncologist. 2010;15:924-34.

10. Johnston S, Pippen Jr J, Pivot X, Lichinitser M, Sadeghi S, Dieras V, et al. Lapatinib combined with letrozole versus letrozole and placebo as first-line therapy for postmenopausal hormone receptor-positive metastatic breast cancer. J Clin Oncol. 2009;27:5538-46.

11. Cardoso F, Costa A, Norton L, Senkus E, Aapro M, Andre F, et al. ESO-ESMO 2nd international consensus guidelines for advanced breast cancer ( $A B C 2)$. Breast. 2014;23:489-502.

12. von Minckwitz G, Schwedler K, Schmidt M, Barinoff J, Mundhenke C, Cufer T, et al. Trastuzumab beyond progression: overall survival analysis of the GBG 26/BIG 3-05 phase III study in HER2-positive breast cancer. Eur J Cancer. 2011;47:2273-81.

13. von Minckwitz G, du Bois A, Schmidt M, Maass N, Cufer T, de Jongh FE, et al. Trastuzumab beyond progression in human epidermal growth factor receptor 2-positive advanced breast cancer: a german breast group 26/ breast international group 03-05 study. J Clin Oncol. 2009;27:1999-2006.

14. Cardoso F, Costa A, Norton L, Senkus E, Aapro M, Andre F, et al. ESO-ESMO 2nd international consensus guidelines for advanced breast cancer (ABC2). Ann Oncol. 2014;25:1871-88.

15. Gradishar WJ, Anderson BO, Blair SL, Burstein HJ, Cyr A, Elias AD, et al. Breast cancer version 3.2014. J Natl Compr Canc Netw. 2014;12:542-90.

16. Dumontet $C$, Jordan MA. Microtubule-binding agents: a dynamic field of cancer therapeutics. Nat Rev Drug Discov. 2010;9:790-803.

17. Slamon DJ, Leyland-Jones B, Shak S, Fuchs H, Paton V, Bajamonde A, et al. Use of chemotherapy plus a monoclonal antibody against HER2 for metastatic breast cancer that overexpresses HER2. N Engl J Med. 2001;344:783-92.

18. Slamon D, Eiermann W, Robert N, Pienkowski T, Martin M, Press M, et al. Adjuvant trastuzumab in HER2-positive breast cancer. N Engl J Med. 2011;365:1273-83

19. Schneeweiss A, Chia S, Hickish T, Harvey V, Eniu A, Hegg R, et al. Pertuzumab plus trastuzumab in combination with standard neoadjuvant anthracyclinecontaining and anthracycline-free chemotherapy regimens in patients with HER2-positive early breast cancer: a randomized phase II cardiac safety study (TRYPHAENA). Ann Oncol. 2013;24:2278-84.

20. Giordano SH, Lin YL, Kuo YF, Hortobagyi GN, Goodwin JS. Decline in the use of anthracyclines for breast cancer. J Clin Oncol. 2012;30:2232-9.

21. Lao J, Madani J, Puertolas T, Alvarez M, Hernandez A, Pazo-Cid R, et al. Liposomal Doxorubicin in the treatment of breast cancer patients: a review. J Drug Deliv. 2013;2013:456409.

22. Montemurro F, Rossi V, Nole F, Redana S, Donadio M, Martinello R, et al. Underuse of anthracyclines in women with HER-2+ advanced breast cancer. Oncologist. 2010;15:665-72.

23. Jones SE, Savin MA, Holmes FA, O'Shaughnessy JA, Blum JL, Vukelja S, et al. Phase III trial comparing doxorubicin plus cyclophosphamide with docetaxel plus cyclophosphamide as adjuvant therapy for operable breast cancer. J Clin Oncol. 2006;24:5381-7.

24. Bayraktar S, Gonzalez-Angulo AM, Lei X, Buzdar AU, Valero V, MelhemBertrandt $A$, et al. Efficacy of neoadjuvant therapy with trastuzumab 
concurrent with anthracycline- and nonanthracycline-based regimens for HER2-positive breast cancer. Cancer. 2012;118:2385-93.

25. Gill SE, Savage K, Wysham WZ, Blackhurst DW, Winter WE, Puls LE. Continuing routine cardiac surveillance in long-term use of pegylated liposomal doxorubicin: is it necessary? Gynecol Oncol. 2013;129:544-7.

26. Keller AM, Mennel RG, Georgoulias VA, Nabholtz JM, Erazo A, Lluch A, et al. Randomized phase III trial of pegylated liposomal doxorubicin versus vinorelbine or mitomycin C plus vinblastine in women with taxanerefractory advanced breast cancer. J Clin Oncol. 2004;22:3893-901.

27. O'Brien ME, Wigler N, Inbar M, Rosso R, Grischke E, Santoro A, et al. Reduced cardiotoxicity and comparable efficacy in a phase III trial of pegylated liposomal doxorubicin $\mathrm{HCl}$ (CAELYX/Doxil) versus conventional doxorubicin for first-line treatment of metastatic breast cancer. Ann Oncol. 2004;15:440-9.

28. Safra T, Muggia F, Jeffers S, Tsao-Wei DD, Groshen S, Lyass O, et al. Pegylated liposomal doxorubicin (doxil): reduced clinical cardiotoxicity in patients reaching or exceeding cumulative doses of $500 \mathrm{mg} / \mathrm{m}^{2}$. Ann Oncol. 2000;11:1029-33.

29. Sparano JA, Makhson AN, Semiglazov VF, Tjulandin SA, Balashova OI Bondarenko IN, et al. Pegylated liposomal doxorubicin plus docetaxel significantly improves time to progression without additive cardiotoxicity compared with docetaxel monotherapy in patients with advanced breast cancer previously treated with neoadjuvant-adjuvant anthracycline therapy: results from a randomized phase III study. J Clin Oncol. 2009;27:4522-9.

30. Chia S, Clemons M, Martin LA, Rodgers A, Gelmon K, Pond GR, et al. Pegylated liposomal doxorubicin and trastuzumab in HER-2 overexpressing metastatic breast cancer: a multicenter phase II trial. J Clin Oncol. 2006;24: 2773-8.

31. Christodoulou C, Kostopoulos I, Kalofonos HP, Lianos E, Bobos M, Briasoulis E, et al. Trastuzumab combined with pegylated liposomal doxorubicin in patients with metastatic breast cancer. phase II Study of the Hellenic Cooperative Oncology Group (HeCOG) with biomarker evaluation Oncology. 2009;76:275-85.

32. Baselga J, Manikhas A, Cortes J, Llombart A, Roman L, Semiglazov VF, et al. Phase III trial of nonpegylated liposomal doxorubicin in combination with trastuzumab and paclitaxel in HER2-positive metastatic breast cancer. Ann Oncol. 2014;25:592-8.

33. Janssen-Cilag International N.V. Caelyx (PEGylated - EU Summary of Product Characteristics. 2015. http://www.ema.europa.eu/docs/en_GB/document_ library/EPAR_-_Product_Information/human/000089/WC500020180.pdf.

34. Hendriks BS, Klinz SG, Reynolds JG, Espelin CW, Gaddy DF, Wickham TJ. Impact of tumor HER2/ERBB2 expression level on HER2-targeted liposomal doxorubicin-mediated drug delivery: multiple low-affinity interactions lead to a threshold effect. Mol Cancer Ther. 2013;12:1816-28.

35. Reynolds JG, Geretti E, Hendriks BS, Lee H, Leonard SC, Klinz SG, et al. HER2targeted liposomal doxorubicin displays enhanced anti-tumorigenic effects without associated cardiotoxicity. Toxicol Appl Pharmacol. 2012;262:1-10.

36. LoRusso P, Krop I, Miller K, Ma C, Siegel BA, Shields A, et al. A phase I study of MM-302, a HER2-targeted PEGylated liposomal doxorubicin, in patients with HER2+ metastatic breast cancer. American Association of Cancer Research Annual Meeting. 2015:abstract CT234.

37. Wolff AC, Hammond ME, Hicks DG, Dowsett M, McShane LM, Allison KH, et al. Recommendations for human epidermal growth factor receptor 2 testing in breast cancer: American Society of Clinical Oncology/College of American Pathologists clinical practice guideline update. J Clin Oncol. 2013;31:3997-4013.

38. Munster P, Krop IE, Miller K, Siegel BA, Shields A, Pampaloni M, et al. Assessment of safety and activity in an expanded phase 1 study of MM-302, a HER2-targeted liposomal doxorubicin, in patients with advanced HER2positive (HER2+) breast cancer. San Antonio Breast Cancer Symposium. 2013;abstract P4-12-29.

39. Fiegl M, Mlineritsch B, Hubalek M, Bartsch R, Pluschnig U, Steger GG. Single-agent pegylated liposomal doxorubicin (PLD) in the treatment of metastatic breast cancer: results of an Austrian observational trial. BMC Cancer. 2011;11:373.

40. Huober J, Fett W, Nusch A, Neise M, Schmidt M, Wischnik A, et al. A multicentric observational trial of pegylated liposomal doxorubicin for metastatic breast cancer. BMC Cancer. 2010;10:2.

41. Andersson M, Lidbrink E, Bjerre $K$, Wist $E$, Enevoldsen $K$, Jensen $A B$, et al. Phase III randomized study comparing docetaxel plus trastuzumab with vinorelbine plus trastuzumab as first-line therapy of metastatic or locally advanced human epidermal growth factor receptor 2-positive breast cancer: the HERNATA study. J Clin Oncol. 2011;29:264-71.

42. Bartsch R, Wenzel C, Gampenrieder SP, Pluschnig U, Altorjai G, Rudas M, et al. Trastuzumab and gemcitabine as salvage therapy in heavily pre-treated patients with metastatic breast cancer. Cancer Chemother Pharmacol. 2008;62:903-10.

43. Lee YR, Huh SJ, Lee DH, Yoon HH, Seol YM, Choi YJ, et al. Phase II study of vinorelbine plus trastuzumab in HER2 overexpressing metastatic breast cancer pretreated with anthracyclines and taxanes. J Breast Cancer. 2011;14:140-6.

44. Yardley DA, Burris III HA, Hanson S, Greco FA, Spigel DR, Barton J, et al. Weekly gemcitabine and trastuzumab in the treatment of patients with HER2overexpressing metastatic breast cancer. Clin Breast Cancer. 2009;9:178-83.

45. Hoffmann La Roche. Xeloda (capecitabine) - US prescribing information. 2015. http://www.accessdata.fda.gov/drugsatfda_docs/label/2015/ 020896s036lbl.pdf.

46. Hoffmann La Roche. Xeloda (capecitabine) - EU Summary of Product Characteristics. 2015. http://www.ema.europa.eu/docs/en_GB/document_ library/EPAR_-_Product_Information/human/000316/WC500058151.pdf.

47. Lilly. Gemzar (gemcitabine) - US prescribing information. 2014. http://www. accessdata.fda.gov/drugsatfda_docs/label/2014/020509s077lbl.pdf.

48. Lilly. Gemzar (gemcitabine) - EU Summary of Product Characteristics. 2014. https://www.medicines.org.uk/emc/medicine/596.

49. Pierre Fabre. Navelbine (vinorelbine) - EU Summary of Product Characteristics. 2011. https://www.medicines.org.uk/emc/medicine/16029.

50. Pierre Fabre. Navelbine (vinorelbine) - US prescribing information. 2014. http:// www.accessdata.fda.gov/drugsatfda docs/label/2014/020388s027lbl.pdf.

51. Michiels S, Pugliano L, Grun D, Barinoff J, Cameron DA, Cobleigh MA, et al. Progression-free survival (PFS) as surrogate endpoint for overall survival (OS) in clinical trials of HER2-targeted agents in HER2-positive metastatic breast cancer (MBC): An individual patient data (IPD) analysis. J Clin Oncol. 2013;31(suppl):abstract 610.

\section{Submit your next manuscript to BioMed Central and we will help you at every step:}

- We accept pre-submission inquiries

- Our selector tool helps you to find the most relevant journal

- We provide round the clock customer support

- Convenient online submission

- Thorough peer review

- Inclusion in PubMed and all major indexing services

- Maximum visibility for your research

Submit your manuscript at www.biomedcentral.com/submit
C Biomed Central 